\title{
Doenças crônicas em crianças e adolescentes: uma revisão bibliográfica
}

Chronic diseases in children and adolescents:

a review of the literature

Martha Cristina Nunes Moreira ${ }^{1}$

Romeu Gomes ${ }^{2}$

Miriam Ribeiro Calheiros de Sá ${ }^{3}$

${ }^{1}$ Departamento de Ensino, Instituto Fernandes Figueira (IFF), Fundação Oswaldo Cruz (Fiocruz). Av. Rui Barbosa $716 / 5^{\circ}$ andar/Saúde e Brincar-Programa de Atenção Integral à Criança Hospitalizada, Flamengo. 22.250-020 Rio de Janeiro RJ Brasil.

moreira@iff.fiocruz.br

${ }^{2}$ Pós-graduação em Saúde da Criança e da Mulher, IFF Fiocruz.

${ }^{3}$ Fisioterapia Motora, IFF

Fiocruz.
Abstract The scope of this article is to analyze papers published between 2003 and 2011 that focus on discussions regarding chronic conditions or chronic diseases in children and adolescents. It combines a methodological review of the literature and thematic analysis of content in order to identify the elements that characterize chronic diseases in children and adolescents and the specificities generated by these chronic conditions. The review of the literature resulted in a description of the series of articles identified by year of publication, country of origin, type of study, population and the chronic condition addressed. Thematic content analysis generated two core themes: Definition of chronic disease and Ways of han dling chronic disease in children and teenagers. The main conclusion reached is that the age transitions when a disease is diagnosed and treated since childhood involve transformations that include changes in health facilities, discharge processes, decision making and networking that include family, hospital, school and institutions that guarantee the child's rights.

Key words Chronic health conditions, Chronic disease, Child, Adolescent, Review of the literature
Resumo $O$ artigo analisa artigos publicados entre 2003 e 2011 enfocando discussões sobre condições crônicas ou doenças crônicas de crianças e adolescentes. Metodologicamente conjuga a revisão de literatura a uma análise de conteúdo temática com vistas a identificar quais os elementos que caracterizam doenças crônicas em crianças e adolescentes e as especificidades geradas por essas condições. A revisão resultou na descrição do conjunto dos artigos, caracterizando-os quanto ano de publicação, país, tipo de estudo, população e condição de cronicidade abordada. A análise de conteúdo temática gerou dois temas: Definição de Doença Crônica $e$ Formas de se lidar com as doenças crônicas em crianças e adolescentes. Destaca-se como conclusão que as transições etárias quando uma doença é diagnosticada e tratada desde a infância, vai passar por transformações que incluem a maneira como se dá o seu fluxo entre os serviços e as mudanças que envolvem processos de alta, de tomada de decisão e de construção de rede que inclua família, hospital, escola e sistema de garantia de direitos.

Palavras-chave Condições crônicas de saúde, Doença crônica, Criança, Adolescente, Revisão bibliográfica 


\section{Introdução}

As condições crônicas de saúde consistem em problemas que demandam tratamento contínuo, de longa duração, exigindo cuidados permanentes ${ }^{1,2}$.

No Brasil, a relevância de se ampliar a discussão acerca das condições crônicas de saúde se assenta ainda na necessidade de encarar a transição epidemiológica da população brasileira, oriunda - dentre outros fatores - do aporte de melhores recursos para diagnóstico e tratamento, de melhoras nos indicadores sociais e de saúde, que modificou a sobrevida, concorrendo com variadas comorbidades associadas na população infanto-juvenil ${ }^{3-5}$.

Essa multiplicidade de agravos geradores de condições crônicas de saúde demanda uma nova lógica de cuidados, tanto em direção a uma nova projeção de práticas em saúde, como para uma reorientação de serviços de saúde, assim como formação e capacitação de profissionais, assinalando a necessidade de investimento em uma nova pediatria ${ }^{4}$. Entretanto, ao observar-se a realidade de atuação de intervenções na área da saúde, observa-se que este campo se ressente de subsídios e bases conceituais, que lhe atribuam maior visibilidade. Essa pode advir da sistematização do conhecimento, enfocando as especificidades das condições crônicas de crianças e adolescentes, bem como as necessidades e demandas geradas.

A partir dessa perspectiva, o estudo objetiva analisar a produção do conhecimento acerca das condições crônicas ou doenças crônicas de crianças e adolescentes, visando contribuir para a sistematização de princípios de cuidados voltados para esses sujeitos.

\section{Material e método}

O desenho metodológico consiste em uma revisão bibliográfica delimitada em artigos científicos no período de oito anos, com início em 2003 e término em 2011. Esse marco inicial se deve ao fato de, nesse ano, a Organização Mundial da Saúde (OMS) definir o "Modelo dos Cuidados Inovadores para as Condições Crônicas” (MCICC) como um suporte intelectual para fazer frente à transição epidemiológica de crescimento das doenças crônicas não só no mundo desenvolvido, mas nos países em desenvolvimento.

A busca foi efetuada na base de dados Medline através do PubMed, e foi antecedida da consulta aos descritores em ciências da saúde, a fim de buscar a definição de doença crônica e de con- dições crônicas de saúde. O segundo termo, condições crônicas de saúde, não foi identificado no DesclMesh. Com relação ao descritor doença crônica e seus correspondentes em inglês e espanhol (chronic disease e enfermedad crónica), a definição segue a partir da delimitação daquilo que poderíamos denominar pela cronicidade do quadro: permanente, com incapacidade residual e uma causalidade baseada em alteração patológica irreversível. Associada à definição circunscreve-se a identidade do sujeito acometido como um paciente que requer atendimento especial, com foco na reabilitação, o que o coloca em posição de um sujeito dependente de supervisão, observação ou cuidado. Como o campo da saúde da criança e do adolescente são nossas interfaces teóricas, associamos ao descritor "doença crônica", as palavras criança e adolescente, como se segue: "Chronic disease"[All Fields] AND "child" [MeSH Terms] OR “adolescent”[MeSH Terms]. Nessa busca, foram encontrados 294 artigos.

$\mathrm{Na}$ leitura dos resumos desses artigos, selecionou-se aqueles que privilegiassem os descritores supracitados de maneira integrada (and). Como esta pesquisa tem um caráter exploratório, considerou-se importante analisar apenas aqueles artigos que abordassem as questões relativas à definição de conceitos de condições crônicas de saúde e às especificidades de crianças e adolescentes que vivem essas condições. Dessa maneira, foram excluídos artigos que abordassem: dor crônica, aspectos econômicos, fatores de risco para doença crônica, uso da tecnologia da informática para informações em saúde, equipamentos, métodos diagnósticos. Com as exclusões, restaram 61 artigos, que constituíram o acervo inicial, o qual foi submetido a uma leitura integral.

Após essa leitura, foram excluídos aqueles que, a despeito de no título ou descritores constar a doença crônica, os mesmos restringiam sua discussão a outros enfoques tais como adoção de crianças com doenças crônicas e intervenções profissionais específicas, com ausência de critérios de desenho de pesquisa. Restaram 40 artigos que constituíram o corpus analítico.

A análise do acervo ocorreu em dois movimentos analíticos. Inicialmente realizou-se uma descrição do conjunto dos artigos, caracterizando-os quanto ano de publicação, país, tipo de estudo, doença ou síndrome abordada e cenário de atendimento.

Em seguida, empreendeu-se uma análise de conteúdo temática ${ }^{6}$ percorrendo as seguintes etapas: leitura exaustiva e crítica do conjunto dos artigos, identificação de núcleos de sentidos nos 
aos países de publicação dos 40 artigos, predominam os da América do norte (USA: $18 \mathrm{e}$ Canadá: 2); seguidos da Europa (Reino Unido: 3, França: 3, Holanda: 2, Suécia: 1 e Alemanha: 1); América do Sul (Brasil: 6 e Argentina: 1) e Austrália (3).

A predominância de produções nos EUA pode se relacionar ao fato de que a discussão sobre modelos de atenção às condições crônicas tenha surgido nesse país e ter sido estimulada pelo MacColl Institute for Healthcare Innovation, responsável por ampla revisão acerca do assunto ${ }^{1,7}$.

Com relação aos métodos empregados nos 40 artigos, temos 20 ensaios teóricos, 04 editoriais, 08 estudos quantitativos e 08 estudos qualitativos. O predomínio de ensaios teóricos aponta para a valorização de processos de revisão conceitual, teórica e de pesquisas que possibilitem melhores evidências e consensos em torno de definições.

Quanto ao ano de publicação, verifica-se o incremento das publicações a partir de 2008. Isso pode se relacionar ao fato de que, na segunda década dos anos 2000, a produção do conhecimento acerca do tema em questão passa a ser influenciada pelo cenário epidemiológico da cronicidade, não só pelo perfil dos adultos e idosos, mas cada vez mais precocemente das crianças, adolescentes e adultos jovens.

Em termos de conteúdos centrais dos artigos, 20 deles tratam de doenças em específico ou de síndromes que levam a morbidades crônicas (Tabela 1), e os outros 20 conseguem alcançar discussões sobre dimensões de vida com a doença crônica e/ou as deficiências e que podem ser exploradas por meio de estudos sobre relações familiares, percepções sobre o adoecimento e qualidade do cuidado.

Dentre as doenças ou síndromes abordadas especificamente pelos estudos e que fazem parte do grupo de patologias que formam os elementos destes artigos, destacam-se as seguintes: asma, epilepsia, leucemia, doenças renais, miopatias, doença reumatoide, encefalopatia crônica da infância.

Em relação aos cenários das pesquisas, observamos que 25 artigos situam as discussões nos ambientes que constituem a vida de crianças e adolescentes com doenças crônicas, ou seja, não somente os diversos níveis da atenção à saúde, mas também escolas, domicílios e, como item a parte, o destaque sobre a forma como os pais se apropriam das recomendações e cuidados (Tabela 2).

Por fim, com relação ao foco de atenção dos artigos, nota-se que 19 apresentam somente crianças como sujeitos da pesquisa, 05 para adolescentes, 05 englobam tanto adolescentes quanto crianças, 07 estão voltados para os pais, 03 com foco nas equipes de saúde e 01 tendo pais e profissionais como sujeitos da pesquisa.

\begin{tabular}{|c|c|c|}
\hline Estudo & Doença ou Síndrome & $\mathbf{N}$ \\
\hline Janse et al. ${ }^{8}$, Marcon et al. ${ }^{9}$, Miller ${ }^{10}$ & Doença Genética & 03 \\
\hline Monje e Almagiá ${ }^{11}$, Alencar e Alencar ${ }^{12}$ & Diabetes & 02 \\
\hline Turkel e $\mathrm{Pao}^{13}$ & Malformação & 01 \\
\hline Towns e Bells ${ }^{14}$ & Fibrose cística & 01 \\
\hline Cooley $^{15}$, Hewitt-Taylor ${ }^{16}$ & Criança com necessidades especiais & 02 \\
\hline Hexem et al. ${ }^{17}$, Christian ${ }^{18}$, Halfon e Newacheck ${ }^{19}$, Silva et al. ${ }^{20}$, & Doença crônica em crianças e/ou & 20 \\
\hline Angstrom-Brannstrom et al. ${ }^{21}$, Mokkink et al. ${ }^{22}$, Janse et al. ${ }^{8}$, Yeo e & adolescentes em geral, ligada a & \\
\hline Sawyer $^{23}$, Freed e Hudson ${ }^{24}$, Lemétayer e Chateaux ${ }^{25}$, Hafez e Miller ${ }^{26}$, & outros debates (experiências & \\
\hline Brown et al. ${ }^{27}$, Almeida et al. ${ }^{28}$, Mack et al..$^{29}$, Schmidt et al. ${ }^{30}$, Orrell- & familiares, percepções sobre o & \\
\hline Valente e Cabana ${ }^{31}$, Taylor et al..$^{32}$, Pedroso e Motta ${ }^{33}$, Sullivan- Bolyai ${ }^{34}$, & adoecimento crônico e/ou as & \\
\hline Dobbie e Mellor ${ }^{35}$, Russo ${ }^{36}$ & deficiências, qualidade do cuidado). & \\
\hline Garcia et al. ${ }^{37}$, Le Blanc et al. ${ }^{38}$, Olson et al. ${ }^{39}$, Nobrega et al. ${ }^{40}$, & Mais de uma doença & 09 \\
\hline \multicolumn{3}{|l|}{ Bouquinet et al. ${ }^{41}$, Chesson et al. ${ }^{42}$, Torpy et al. ${ }^{43}$, Roizenet al. ${ }^{44}$, Miller ${ }^{45}$} \\
\hline \multirow[t]{2}{*}{ Oeseburg et al. ${ }^{46}$, Herzer et al. ${ }^{47}$} & Retardo mental & 02 \\
\hline & & 40 \\
\hline
\end{tabular}




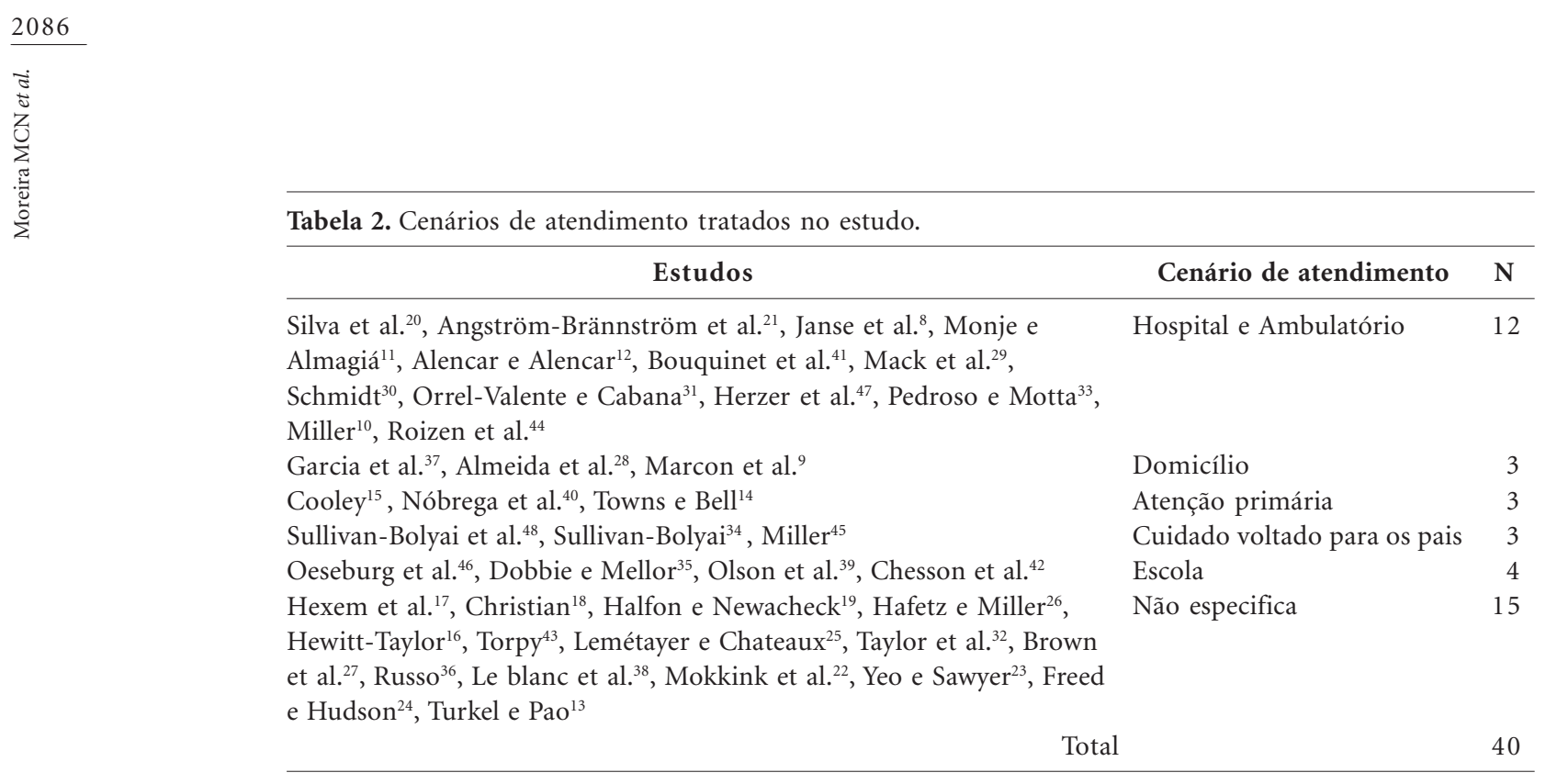

\section{A tematização dos conteúdos dos artigos}

A partir da análise foram identificadas duas temáticas: Definição de Doença Crônica e Formas de se lidar com as doenças crônicas em crianças e adolescentes.

Em relação à primeira temática - Definição de Doença Crônica - observam-se cinco núcleos de sentidos que se desprendem dos conteúdos dos artigos e que podem ser ilustrados por uma árvore de ideias (Figura 1).

$\mathrm{O}$ primeiro núcleo diz respeito às definições que se estruturam a partir da necessidade de suporte para o desenvolvimento das funções motoras e comunicacionais contribuindo para a interação com o meio, com as pessoas e o reconhecimento de suas expressões afetivas, desejos e vontades $^{17-21,37,38}$. Neste núcleo, identificam-se os conhecimentos e as técnicas que possam facilitar a mobilidade, a interação com o meio através da criação de códigos linguísticos adaptados, uso de tecnologia assistiva.

O segundo núcleo de sentido se refere ao fato de autores ${ }^{17-19,21,38}$ definirem doença crônica como aquela que demanda cuidados que envolvem a utilização de recursos tecnológicos de suporte à vida. Compõem esses recursos: medicamentos, dietas especiais, ventilação mecânica invasiva ou não invasiva, nutrição parenteral, oxigenoterapia, cateter totalmente implantado.

Como terceiro núcleo de sentido, destaca-se a definição de doenças crônicas a partir da permanência e da recorrência de episódios da doença, considerado meses de vida ${ }^{8,11-13,15,16,19-26,37-40}$.

A presença de comorbidades associadas constitui-se no quarto núcleo de sentido encontrado no conjunto dos artigos ${ }^{11-13,15,16,19,20,22,24,25,27-30,37-}$ $39,41,43,46,48$. Essa definição caracteriza a doença crônica como um quadro complexo de agravos.

Por último, o quinto núcleo de sentido se centra na ideia de que a doença crônica envolve a limitação da funcionalidade com comprometimento do desenvolvimento global e qualidade de vida ${ }^{8,9,11,13,15,16,19,23,25,30-32,37-41,43,46-48}$.

A árvore de ideias da primeira temática ( $\mathrm{Fi}$ gura 1) é fruto da análise e interpretação de artigos que destacam definições e/ou tentativas de especificar as características das doenças crônicas quando as mesmas acometem crianças e adolescentes. Nos artigos reunidos destaca-se na constelação de definições a centralidade da doença na vida, com o comprometimento de funções vitais, desde motoras até fisiológicas. Ou seja, a definição surge ancorada nos déficits ou ainda nas necessidades que estão relacionadas novamente à dependência. Assim, observou-se que, em geral, as definições centram-se numa doença sem sujeito ou em um deficitário e dependente. Com isso não se quer desvalorizar as definições, mas apenas chamar a atenção para a necessidade de não descontextualizar a doença, do sujeito com a doença. No caso de situações de adoecimento em crianças e adolescentes, a própria singularidade do desenvolvimento e crescimento desses sujeitos revela uma necessidade de ampliar definições que articulem dimensões ampliadas da vida. Destaca-se ainda a ideia de complexidade relacionada a aspectos clínicos, da biomecânica do corpo, perdendo de vista, mais uma vez, a noção ampliada da vida como sistema e do corpo não somente como base biológica, mas como suporte para a dimensão simbóli- 


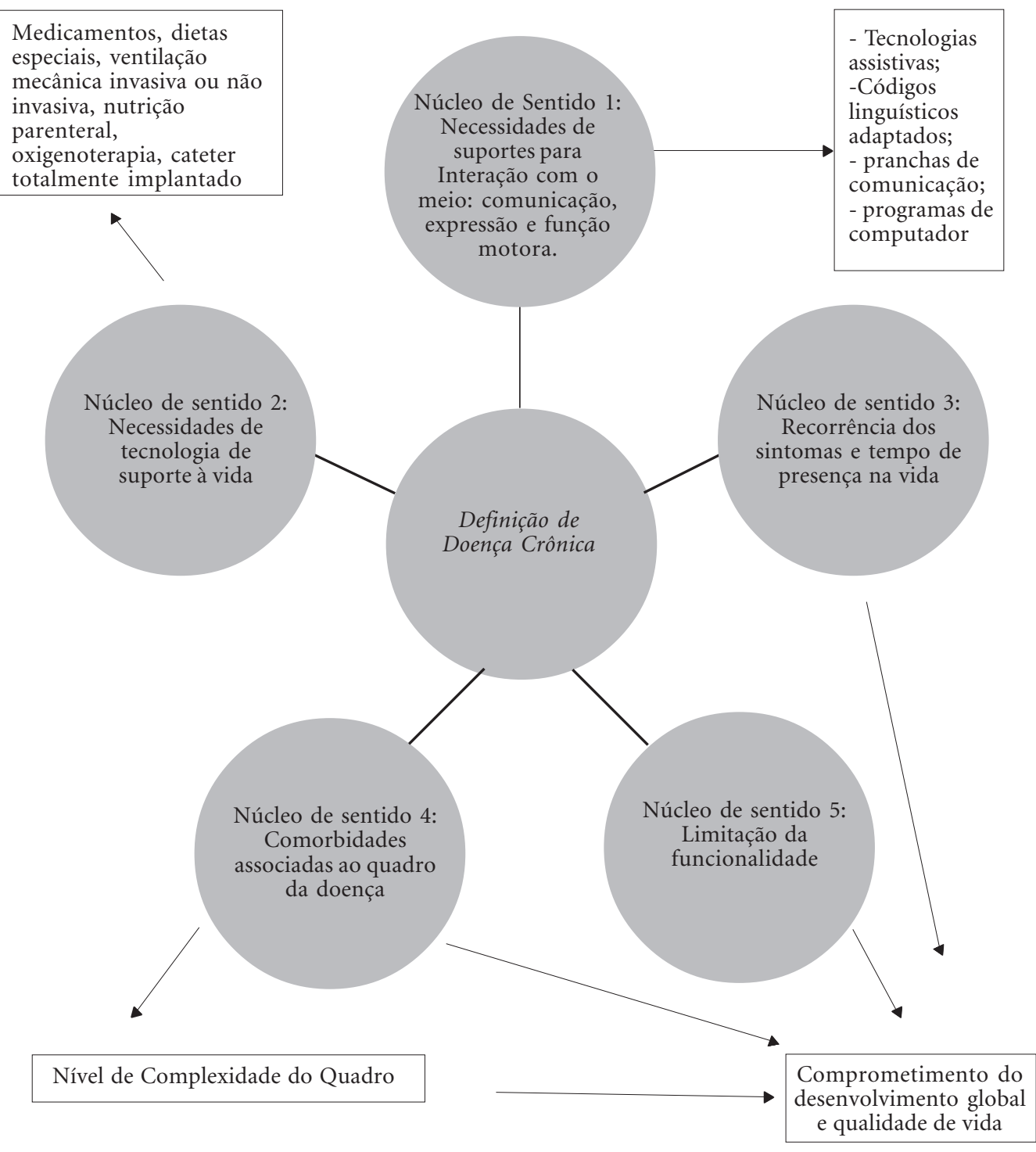

Figura 1. Descrição da seleção dos estudos.

ca, das representações, interpretações e valores sobre a existência. E mais uma vez, torna-se imperativo situar qual o sujeito que adoece cronicamente, se adulto, criança ou adolescente, na definição de um quadro crônico e de suas consequências.

A segunda temática - Formas de se Lidar com as Doenças Crônicas - se resume em dois núcleos de sentido, ilustrados na árvore de ideias a seguir (Figura 2).

O primeiro núcleo se refere a propostas de se promover o protagonismo da criança e do adolescente no cuidado da saúde, incluindo-se estratégias lúdicas na comunicação adaptada de notí- cias de acordo com a faixa etária e a participação ativa no processo decisório de seu tratamento assim como na transição entre os serviços ${ }^{10-}$ 14,19,21,23-25,29,31-35,41,42,45,47. Já o segundo núcleo de sentido se relaciona à defesa da ideia de que para se lidar com as condições crônicas se faz necessário a criação de redes de apoio, tais como sistemas organizacionais e de suporte mediante os quais os cuidados são empreendidos ${ }^{8-15,17,18,21,23-}$ 25,27,29-37,39-41,44,45,47,48.

No que se refere à segunda árvore de ideias (Figura 2), destaca-se que no momento em que se assume a necessidade de situar a doença na vida, ao defini-la, são alcançados seus sujeitos, 


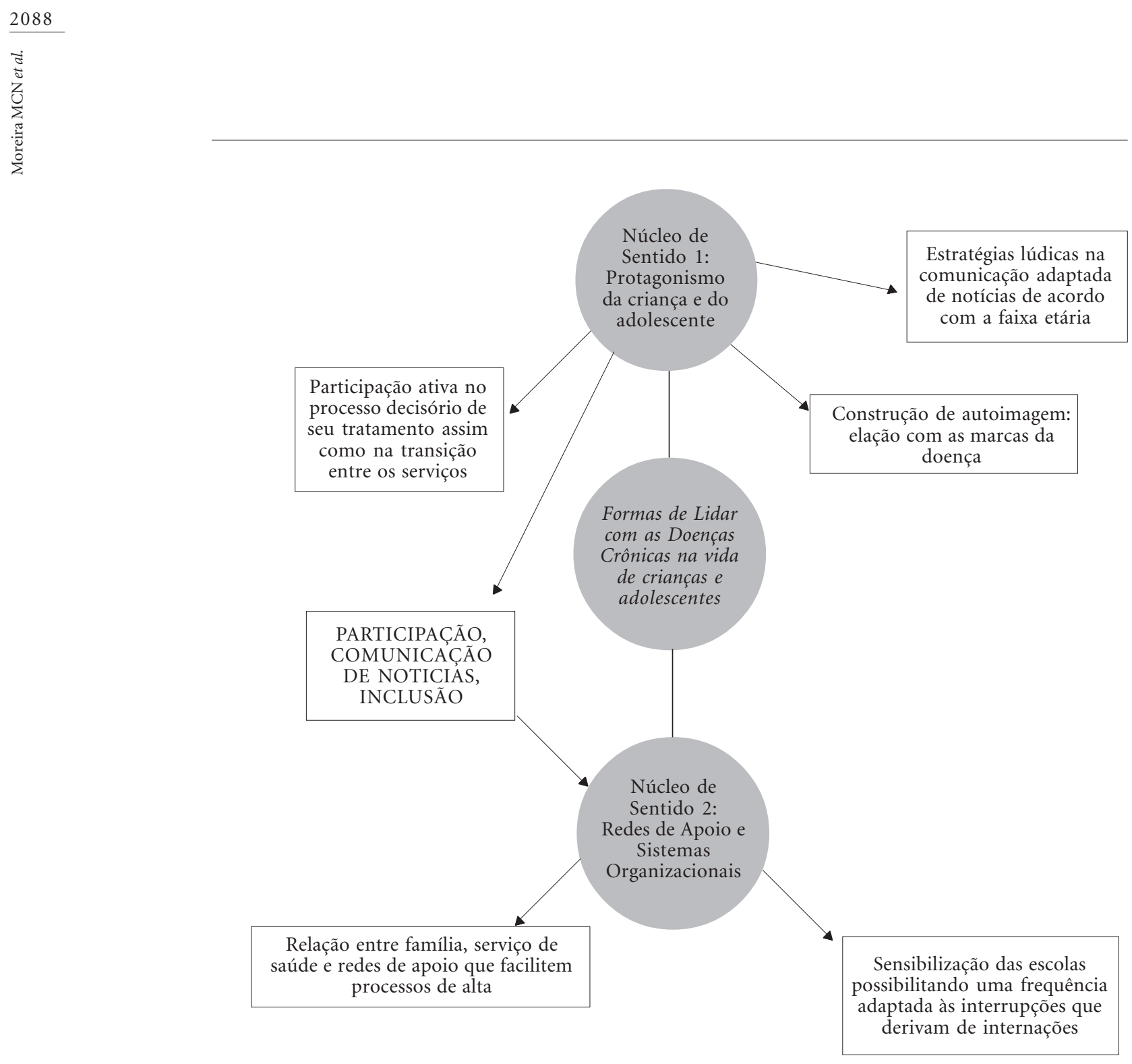

Figura 2. Árvore de ideias relacionadas às Formas de se lidar com as doenças crônicas em crianças e adolescentes.

que no caso de crianças e adolescentes com doenças crônicas ganham centralidade as ideias de comunicação de noticias, participação e inclusão. E nessa direção alcança-se a valorização de mecanismos facilitadores e já reconhecidos como promotores do protagonismo infanto-juvenil: as intervenções lúdicas. Outro aspecto bem característico do reconhecimento de crianças e jovens na definição diz respeito à inclusão nas instituições escolares, e no esclarecimento de seus atores - professores, diretores, colegas - das possibilidades e limites que cada doença crônica, situada na vida particular do sujeito, pode causar.

\section{Discussão dos Resultados}

Emergem como elementos centrais da primeira temática aqueles que delimitam a doença pela perspectiva médica com um recorte vinculado às alterações que diferenciam a dimensão física da emocional $^{15,30,43}$, enfocando a definição da doença crônica aos seus mecanismos de explicação e suas causas $^{49}$. Nesses artigos, o não reconhecimento das possíveis diferenças entre as doenças crônicas da infância (asma, fibrose cística, obesidade, desnutrição, alterações do desenvolvimento, paralisia cerebral, consequências da prematuridade e transtornos mentais) pode colocar ao menos dois problemas: 1) a desconsideração da diversidade dos agravos e/ou comorbidades associadas que 
reduz as singularidades a uma categoria única $\mathrm{e}$ geral, a de "doença crônica", sem problematizar as peculiaridades de cada uma e as diferentes evoluções clínicas; 2) a não consideração de que essa generalidade pode comprometer o plano das identidades pessoais e da organização das famílias. Nesse caso, o diagnóstico que nomeia e delimita e, portanto, que gera condições para mecanismos de identificação, pode gerar condições para a formação de grupos de apoio.

Nos artigos revisados ${ }^{15,30,43}$ identifica-se ainda uma outra redução: a do cuidado a essas doenças crônicas na infância a um nível de maior complexidade do sistema de saúde, desconsiderando a possibilidade de estratégias de promoção e prevenção em saúde mesmo quando já estão instaladas. Para tanto justificam que a complexidade das alterações (físicas, emocionais, desenvolvimento, comportamento) extrapola o cuidado domiciliar primário. E, ainda, essa perspectiva compromete o entendimento de ações articuladas, onde a estratégia de saúde da família/medicina de família pode vir a desenvolver ações com crianças com necessidades especiais/ condições crônicas.

Além das reduções nas definições da doença em questão, há ideias que ampliam a discussão. Uma delas está presente em artigos que evocam dados epidemiológicos e avaliações focadas em perspectivas de análises de rede, organização de processos de comunicação, tomada de decisão e alta $^{10,12,17,18,22,23,27,31,37}$. Um dos artigos ${ }^{33}$ aciona o conceito de vulnerabilidades socioeconômicas com base numa perspectiva ecológica, tendo como sujeitos de pesquisa enfermeiras que atuavam em unidades de internação pediátricas. Ainda que esses artigos ampliem a discussão, persiste a nomeação da condição crônica como complexa, fazendo equivaler a complexidade a uma leitura biomédica, onde as morbidades múltiplas desencadeiam um esfacelamento da experiência do adoecer em especialidades, de base multidisciplinar. Essa multidisciplinaridade, apenas como um aglomerado de profissões trabalhando sob a coordenação médica não faz aparecer uma integração entre disciplinas. Outro grupo de arti$\operatorname{gos}^{16,19,20,40}$ destaca a temporalidade dos sintomas, o nível do comprometimento das restrições funcionais e de vida associado ao uso dos serviços para definir uma doença crônica bem de acordo com as definições da OMS ${ }^{1}$. Alem de usar esses parâmetros, valorizam a lógica do modelo social da deficiência que desconstrói uma suposta desvantagem natural atribuída ao deficiente, discutindo que sobre as marcas da deficiência incidem opressões que os discriminam. Uma leitura sociológica e política alimenta essa discussão contribuindo para a valorização da autoorganização das pessoas deficientes em movimentos sociais no escopo da identidade de minorias. Ao mesmo tempo, esses autores recuperam a transição epidemiológica e dos indicadores sociais na relação com as transformações na chamada "ecologia social da infância". Para defini-la retomam a abordagem ecossistêmica na relação entre os determinantes sociais da saúde e as situações de adoecimento. No caso, apontam a exposição das crianças a altos níveis de toxicidade, ao stress e ao sedentarismo associada às mudanças no estilo de vida, dentre elas aquelas relacionadas à ingestão de altas taxas calóricas.

Por fim, ainda no interior da primeira temática, identifica-se o uso das definições de qualidade de vida ${ }^{8,29}$, de modo a problematizar as percepções sobre tratamento e comunicação, ou ainda, com a preocupação de validar tais escalas considerando como sujeitos os responsáveis e também as crianças e adolescentes ${ }^{44}$. Diferentemente de crianças com outras doenças, aquelas com condições crônicas de saúde e seus pais acumulam conhecimento pela experiência com o sistema de cuidados, e as variáveis sociodemográficas devem ser incluídas nas considerações sobre a vida mediada pela doença. Por isso, elas não apresentariam necessidades complexas de informações, ou seja, o tempo de vivência e convivência com a doença, ou até mesmo o fato de ser uma criança sem doença crônica, mas com histórico de internação em UTI, influencia a percepção do estado de saúde e intervém em sua qualidade de vida. Já em Towns e Bell ${ }^{14}$, Herzer et al. ${ }^{47}$, Freed e Hudson ${ }^{24}$, Miller ${ }^{45}$ e Oeseburg et al ${ }^{46}$, são valorizados os conceitos de ciclo de vida e estratificação de faixa etária na definição de doença crônica.

A segunda temática, formas de se lidar com a doença crônica, se volta para os papéis sociais e as práticas de aconselhamento de atores-chave nas organizações escolares e de atenção à saúde, mediadoras na formação e nos cuidados com crianças e adolescentes. Assim, são focalizadas a estratificação das doenças e a especificidade do campo de pesquisa, relacionado à organização escolar e aos processos de inclusão de crianças com condições crônicas ${ }^{39}$; acionando outros espaços sociais ocupados por crianças e adolescentes com condições crônicas, tais como, escola e família e as expressões sobre processos de alta e de construção de autoimagem ${ }^{11,13,21,25,28,34 \text { - }}$ $36,38,39,41,42,48$. Sob a influência da psicologia comportamental e da sociologia dos papéis sociais, 
alguns conceitos são recorrentes, tais como, coping, papéis sociais, adaptação/inclusão, aconselhamento, autoestima, imagem corporal, estresse, e ainda alguns quadros associados, tais como, o da síndrome de Burnout, para afirmar a especificidade e a relevância da doença crônica como um fator que intervém no desenvolvimento.

O tema da comunicação, seja ela intrafamiliar ou das equipes com as famílias ou crianças ou adolescentes, se destaca sobre como incluir, em que momento e com que apoios a criança em processo de crescimento e que chega à adolescência no seu tratamento de saúde ${ }^{26}$. A relação entre autoeficácia e autoconceito media a interação entre desenvolvimento individual e interação com o ambiente social. Valorizam a perspectiva de que ter conhecimento acerca de si mesmo e do mundo externo contribui para o desenvolvimento de competências, e ainda para a aquisição de um sentido de eficácia pessoal. Essa visão utilitarista e funcional do conhecimento os aproxima de uma leitura reducionista, a qual pode ser alvo de críticas na medida em que o conhecimento/informação, por si só, não garante mudanças de comportamento $^{28}$, ainda mais quando os sentimentos de insegurança e a realidade socioeconômica marcada pela pobreza impossibilita o cumprimento de planos e acordos. Nesse marco analítico há uma comunicação entre definições já anteriormente identificadas, como por exemplo, as condições crônicas em crianças se revelam como promotoras de cuidados complexos. Nesses artigos, outros atores tornam-se sujeitos de pesquisa, no caso os professores $^{39}$, destacando que em relação a crianças e adolescentes com AIDS e epilepsias a percepção desses profissionais está marcada pelos pré-conceitos. Por exemplo, a imagem de que a epilepsia leva a uma incapacidade neurológica e a alterações comportamentais que mobilizam a reorganização da estrutura escolar.

O envolvimento de várias categorias de profissionais é importante, principalmente se esses conseguem perceber as crianças e os adolescentes em condições crônicas para além de avaliações comportamentais. Ou seja, é importante que os profissionais fiquem atentos não só aos riscos que comportamentos podem oferecer aos grupos de convivência, mas também prestem atenção ao sofrimento que pode ser expresso na experiência de gerenciamento da condição crônica, não passando despercebidas as capacidades de crianças e adolescentes.

Com relação ao reconhecimento das crianças e de suas expressões, Lemetayer ${ }^{25}$ assinala que conhecer suas estratégias de adaptação pode sig- nificar enfrentar as relações entre situações negativas e influências na aderência ao tratamento. Significativo refletir aqui sobre as relações que as crianças estabelecem com seus cuidadores e suas sensibilidades em identificar aspectos de atenção, escuta, capacidade de oferecer acolhimento e como estes podem estabelecer circuitos de troca positiva durante processos de tratamento e hospitalização.

No conjunto dos estudos revisados, há alguns que consideram crianças e adolescentes como sujeitos que reconhecem situações negativas e positivas, e esse reconhecimento pode promover estratégias de adaptação à doença que vão influenciar no processo de negociação de estratégias de cuidado para esse segmento. Citamos aqui o reconhecimento do brincar como mediador para os tratamentos de saúde e a necessidade da presença dos pais durante hospitalizações e tratamentos ${ }^{25,41}$.

Assim, esses artigos contribuem para refletir sobre as seguintes especificidades: a) crianças com necessidades de cuidados especiais de saúde necessitam ser recebidas para tratamentos a partir das suas necessidades e das demandas que geram, em virtude da condição de saúde que as marca, e de serem sujeitos em desenvolvimento. Assim os marcadores delimitariam o quanto, como e onde precisariam de medicalização, monitorização, suporte tecnológico e cuidados contínuos. Daí definirem as condições complexas como aquelas referidas às necessidades continuadas de saúde: longo tempo de oxigenoterapia ou ventilação assistida, alimentação e com limitações motoras e cognitivas (escuta, aprendizagem, mobilidade e comunicação). Ou seja, essa definição é gerada a partir da descrição de quais necessidades de suporte essa criança possui; b) crianças são diferentes de adolescentes, e quanto maior a idade maior o acúmulo de repertório que lhes permite desenvolver estratégias mais apoiadoras no processo de viver a doença ${ }^{11,12,23}$; c) no que se refere ainda aos adolescentes, ganha destaque para eles o fato de que precisam comunicar-se com seus médicos ${ }^{29}$.

No que se refere ao avanço na perspectiva de um sistema de atenção a saúde, esse grupo de artigos apontam para a complementaridade entre as medidas para monitoramento e medição da eficácia da prática clínica e os indicadores baseados na clínica convencional (análises laboratorial e radiológica). Nesse cruzamento pode ser possível a avaliação do sucesso e bem-estar do paciente, buscando para tanto suporte nos protocolos de qualidade de vida. 
No caso das experiências das mães, Almeida et al. ${ }^{28}$ ressaltam a experiência de viver em domicilio e de estabelecer relações de cuidado nesse espaço. Identificam as crianças com doenças crônicas como complexas, cujos cuidados também são assim qualificados. Ganha destaque a singularidade do significado da alta para o domicilio como um processo digno de ser vivido com insegurança e medo. Diferentemente do processo de alta de crianças cuja existência não é marcada pela cronicidade. As orientações para alta, muitas vezes dependentes de insumos e materiais como luvas e seringas, tornam-se um desafio para essa parcela da população se ainda houver a marca da pobreza.

\section{Conclusão}

A revisão de literatura cientifica possibilitou reconhecer que - muito embora a doença esteja colocada como descritor - os artigos elaboram reflexões, discussões, conhecimentos tendo como perspectiva a necessidade de gerenciar a doença como uma condição mediadora da vida e do desenvolvimento de crianças e adolescentes até a chegada a vida adulta.

Dessa forma, a revisão empreendida nos possibilita identificar respostas relacionadas ao reconhecimento de crianças e adolescentes com condições crônicas como sujeitos cujas experiências precisam ser integradas pelos adultos de referência em seus cuidados - sejam eles a equipe, os familiares ou os educadores. Nesse sentido, a dimensão da comunicação nos processos de transição etária, considerando as particularidades dos ciclos de vida, é considerada. A ideia é que essas transições merecem ser melhor abordadas caso se ajustem protocolos às perspectivas dos valores, vínculos e emoções que fazem parte dos processos.

O reconhecimento ainda dessa clientela vem no deslocamento da visão adultocêntrica que já convive com concepções de que estratégias adequadas a cada faixa etária merecem ser consideradas. Nessa direção, ações mediadas pelo brincar, como estratégia de saúde específica para criança, são apontadas e reconhecidas. A perspectiva de um sistema que precisa construir-se em rede fica muito flagrante na medida em que no campo da saúde da criança e do adolescente a particularidade das condições crônicas acentua ainda mais as necessidades de relações intersetoriais. As análise relacionais - pautadas na perspectiva de estudos sobre redes de atenção à saúde - merecem ser acionadas como estratégias metodológicas para fomentar pesquisas nesse campo.

A condição crônica de saúde de crianças e adolescentes se define não somente a partir do tempo de adoecimento e das frequências aos serviços de saúde, ou ainda pelo impedimento da rotina. No caso desses sujeitos, destaca-se a consideração de que as transições etárias quando uma doença é diagnosticada e tratada desde a infância, vai passar por transformações que incluem a maneira como se dá o seu fluxo entre os serviços e as mudanças, as quais englobam processos de alta, de tomada de decisão e construção de rede que abranja família, hospital, escola e sistema de garantia de direitos. Isso se torna importante em virtude de predominar na revisão empreendia a ausência de consenso sobre quando e como começar a transição do tratamento das crianças para o adolescente, e daí para a idade adulta. Essa discussão acaba por colocar em questão a capacidade de organização do sistema de saúde como uma rede integrada.

A partir da revisão empreendida pode-se apontar algumas características que contribuiriam para uma definição sobre condição crônica em crianças e adolescentes:

. Permanente na criança por mais de 03 meses, ou ocorreu um episódio clínico 03 vezes ou mais no último ano e provavelmente vai reincidir; qual a duração e as limitações funcionais impostas, a duração e a necessidade de adaptações;

- Comprometimento das dimensões de sociabilidade específicas da infância, as quais pode se definir, além dos recortes clínicos, por: dias de ausência na escola, limitações de atividades cotidianas e de vida diária para a criança, marcos do desenvolvimento;

- Necessita de apoio para as funções humanas de interação, comunicação, expressão e necessidades de suporte tecnológico à vida;

- Caracterizada pela vulnerabilidade que pode associar alterações nas condições físicas, emocionais, de desenvolvimento e comportamentais e que necessitem de cuidados de serviços de saúde, além dos usuais, e de domiciliares primários.

. Possível presença de relações de associação entre fatores genéticos/familiares e fatores pré e pós-neonatais.

Aponta-se como limites do presente artigo aqueles voltados a duas ordens de questões: 1) inerente a uma opção metodológica no recorte temporal acionado para a seleção de artigos. $\mathrm{Ou}$ seja, não consideramos produções anteriores a 2003 por assumirmos que a revisão da definição e do apontamento de inovações no cuidado à 
condição crônica pela OMS nesse ano se torna um marco político, no reconhecimento por uma agência internacional; 2) com relação às fontes, foram escolhidos os artigos indexados nas bases de consultas, excluindo teses e livros.

\section{Colaboradores}

MCN Moreira participou da concepção e delineamento do artigo, elaboração da metodologia, análise e interpretação dos dados, redação final. R Gomes participou da concepção e delineamento do artigo e revisão crítica. MRC Sá participou da pesquisa, concepção do artigo, análise e interpretação dos dados, redação final.

\section{Referências}

1. Organização Mundial da Saúde (OMS). Cuidados inovadores para condições crônicas: componentes estruturais de ação: relatório mundial. Brasília: OMS; 2003.

2. Ben Shlomo Y, Kuh D. A life course approach to chronic disease epidemiology: conceptual models, empirical challenges, and interdisciplinary perspectives. Int J Epidemiol 2002; 31(2):285-293.

3. Wagner EH. Chronic disease management: what will take to improve care for chronic illness? Eff Clin Pract 1998; 1(1):2-4

4. Lyra GV, Nations MK, Catrib AMF. Cronicidade e cuidados de saúde: o que a antropologia da saúde tem a nos ensinar. Texto Contexto Enfermagem 2004; 13(1):147-155.

5. Moreira MEL, Goldani MZ A criança é o pai do homem: novos desafios para a área de saúde da criança. Cien Saude Colet 2010; 15(2):321-327.

6. Costa MTF, Gomes MA, Pinto M. Dependência crônica de ventilação pulmonar mecânica na assistência pediátrica: um debate necessário para o SUS. Cien Saude Colet 2011; 16(10):4147-4159.

7. Bardin L. Análise de Conteúdo. Lisboa; Edições 70; 2009.

8. Janse AJ, Sinnema G, Uiterwaal CSPM, Kimpen JLL, Gemke RJBJ. Quality of life in chronic illness: perceptions of parents and paediatricians. Arch Dis Child 2005; 90(5):486-491.

9. Marcon SS, Sassá AH, Soares NTI, Molina RCM. Dificuldades e conflitos enfrentados pela família no cuidado cotidiano a uma criança com doença crônica. Cienc Cuid Saude 2007; 6(Supl. 2):411-419.

10. Miller AR, Condin CJ, McKellin WH, Shaw N, Klassen AF, Sheps S. Continuity of care for children with complex chronic health conditions: parents' perspectives. BMC Health Services Research 2009; 9:242.

11. Monje MJA, Almagiá EB. Autoeficacia, Apoyo Social y Calidad de Vida en Adolescentes com Enfermedades Crónicas. Terapia Psicológica 2008; 26(2): 165-172.

12. Alencar DC, Alencar AMPG. O papel da família na adaptação do adolescente diabético. Rev Rene 2009; 10(1):1-165. 
13. Turkel S, Pao M. Late Consequences of Pediatric Chronic Illness. Psychiatr Clin North Am 2007; 30(4): 819-835.

14. Towns SJ, Bell SC. Transition of adolescents with cystic ûbrosis from paediatric to adult care. The Clinical Respiratory Journal 2011; 5(2):64-75.

15. Cooley WC. Redefining primary pediatric care for children with special health care needs: the primary care medical home. Curr Opin Pediatr 2004; 16(6): 689-692.

16. Hewitt-Taylor J. Supporting children with complex health needs. Nursing Standard 2010; 24(19):50-56.

17. Hexem KR, Bosk AM, Feudtner C. The dynamic system of parental work of care for children with special health care needs: a conceptual model to guide quality improvement efforts. BMC Pediatr 2011; 11:95.

18. Christian BJ. Challenger for parents and families: demands of caregiving of children with chronic condition. J Pediatr Nurs 2010; 25(4):299-301.

19. Halfon N, Newacheck PW. Evolving notions of childhood chronic illness. JAMA 2010; 303(7):665666.

20. Silva JB, Kirschbaum DIR, Oliveira I. Significado atribuído pelo enfermeiro ao cuidado prestado à criança doente crônica hospitalizada acompanhada de familiar. Revista Gaúcha de Enfermagem 2007; 28(2):250-259.

21. Angström-Brännström C, Norberg A, Jansson L. Narratives of children with chronic illness about being comforted. J Pediatr Nurs 2008; 23(4):310316.

22. Mokkink LB, van der Lee JH, Grootenhuis MA, Offringa M, Heymans HAS. Defining chronic diseases and health conditions in childhood $(0-18$ years of age): national consensus in the Netherlands. Eur J Pediatr 2008; 167(12):1441-1447.

23. Yeo M, Sawyer S. Chronic illness and disability. BMJ 2005; 330:721.

24. Freed GL, Hudson EJ. Transitioning children with chronic diseases to adult care: current knowledge, practices, and directions. J Pediatr 2006;148(6):824827.
25. Lemétayer F, Chateaux V. Les instruments de mesure des strategies adaptatives des enfants face a ùne maladie chronique: une revue de la literature. Arch Pédiatr 2008; 15(2):162-169.

26. Hafetz J, Miller VA. Child and parent perceptions of monitoring in chronic illness management: a qualitative study. Child Care Health Dev 2010; 36(5): 655-662.

27. Brown RT, Wiener L, Kupst MJ, Brennan T, Behrman R, Compas BE, David Elkin T, Fairclough DL, Friebert S, Katz E, Kazak AE, Madan-Swain A, Mansfield N, Mullins LL, Noll R, Patenaude AF, Phipps S, Sahler OJ, Sourkes B, Zeltzer L. Single parents of children with chronic illness: an understudied phenomenon. J Pediatr Psychol 2008; 33(4):408-421.

28. Almeida MI, Molina RCM, Vieira TMM, Higarashi $\mathrm{IH}$, Marcon SS. O ser mãe de uma criança com doença crônica: realizando cuidados complexos. Esc Anna Nery R Enferm 2006; 10(1):36-46.

29. Mack JW, Co JPT, Goldmann DA, Weeks JC, Cleary PD. Quality of Health Care for Children: Role of Health and Chronic Illness in Inpatient Care Experiences. Arch Pediatr Adolesc Med 2007; 161(9):828834.

30. Schmidt S, Thyen U, Petersen C, Bullinger M. The performance of screener to identify children with special health care needs in a European sample of children with chronic conditions. Eur J Pediatr 2004. 163(9):517-523

31. Orrell-Valente JK, Cabana MD. The apple doesn't fall far from the tree': the role of parents in chronic disease self-management. Curr Opin Pediatr 2008; 20(6):703-704.

32. Taylor RM, Gibson F, Franck LS. The experience of living with a chronic illness during adolescence: a critical review of the literature. J Clin Nurs 2008; 17(23):3083-3091.

33. Pedroso MLR, Motta MGC. Vulnerabilidades socioeconômicas e o cotidiano da assistência de enfermagem pediátrica: relato de enfermeiras. Anna Nery Rev Enferm 2010; 14(2):293-300. 
34. Sullivan-Bolyai S, Sadler L, Knafl KA, Gilliss CL. Great expectations: a position description for parents as caregivers: Part I. Pediatr Nurs 2003; 29(6): 457-461.

35. Dobbie M, Mellor D. Chronic illness and its impact: considerations for psychologists. Psychol Health Med 2008; 13(5):583-590.

36. Russo VL. Parents of chronically ill children: exploring the experiences of partnered and unpartnered parents.2006. Honors Junior/Senior Projects. Paper 36.

37. Garcia DSO, Gatti G, Costa DL. Visitas domiciliares a crianças com doenças crônicas: influência na formação do estudante de medicina. O Mundo da Saúde 2010; 34(3):327-335.

38. LeBlanc LA, Goldsmith T, Patel DR. Behavioral aspects of chronic illness in children and adolescents. Pediatr Clin N Am 2003; 50(4):859-878.

39. Olson AL, Seidler AB, Goodman D, Gaelic S, Nordgren R. School Professionals' Perceptions About the Impact of Chronic Illness in the Classroom. Arch Pediatr Adolesc Med 2004; 158(1):53-58.

40. Nóbrega VM, Collet N, Silva KL, Coutinho SED. Rede e apoio social das famílias de crianças em condição crônica. Rev. Eletr. Enf. [Internet] 2010; 12(3):431-440. [acessado 2014 maio 15]. Disponível em: http://www.fen.ufg.br/revista/v12/n3/v12 n3a03.htm. doi: 10.5216/ree.v12i3.7566.

41. Bouquinet E, Balestra J, Bismuth E, Bruna A.-L, Gallet S, Harvet G, Jean S, Jousselme C. Maladie chronique et enfance: image du corps, enjeux psychiques et alliance thérapeutique. Arch Pédiatr 2008; 15(4):462-468.

42. Chesson RA, Chisholmb D, Zawc W. Counseling children with chronic physical illness. Patient Education and Counseling 2004; 55(3):331-338.

43. Torpy JM, Campbell A, Glass RM. Chronic disease of children. JAMA 2010; 303(7):682-623.
44. Roizen M, Figueroa C, Salvia 1. Health-related quality of life in children with chronic diseases; comparison of the view of the children, their parents and their physicians. Arch. argent. Pediatr. 2007; 105(4):305-313

45. Miller VA. Parent-Child Collaborative Decision Making for the Management of Chronic Illness: A Qualitative Analysis. Fam Syst Health 2009; 27(3):249266

46. Oeseburg B, Jansen DEMC, Dijkstra GJ, Groothoff JW, Reijneveld SA. Prevalence of chronic diseases in adolescents with intellectual disability. Res Dev Disabil 2010; 31(3):698-704.

47. Herzer M, Goebel J, Cortina S. Transitioning cognitively impaired young patients with special health needs to adult-oriented care: collaboration between medical providers and pediatric psychologists. Curr Opin Pediatr 2010; 22(5):668-672.

48. Sullivan-Bolyai S, Knafl KA, Sadler L, Gilliss CL. Great expectations: a position description for parents as caregivers: Part II. Pediatr Nurs 2004; 30(1):52-56.

49. Camargo Júnior KR. A biomedicina. Physis 2005; 15(Supl.):177-201.

Artigo apresentado em 19/10/2013

Aprovado em 16/11/2013

Versão final apresentada em 22/11/2013 